\title{
Botulinum Toxin for Benign Essential Blepharospasm, Hemifacial Spasm and Age-Related Lower Eyelid Entropion
}

\author{
Jean Carruthers and Harrison A. Stubbs
}

\begin{abstract}
Purified botulinum A exotoxin was used in the treatment of forty seven patients with benign essential blepharospasm, 11 patients with hemifacial spasm and 2 patients with age-related entropion. The treatment was effective in all three groups for an average of 3-4 months when symptoms recurred and repeated chemodenervation with toxin was needed. The commonest complication was transient ptosis with an overall frequency of $7.8 \%$. This incidence increased to $11.1 \%$ with toxin doses higher than 25 units per orbicularis. The treatment was well accepted by the patients, who were subsequently able to return to pre-blepharospasm lifestyles.
\end{abstract}

RÉSUMÉ: Traitement au moyen de la toxine botulique du blépharospasme bénin essentiel, du spasme de l'hémiface et de l'entropion de la paupière inférieure en rapport avec l'âge Nous avons utilisé l'exotoxine botulique A purifiée pour traiter quarante-sept patients avec blépharospasme essentiel bénin, onze patients avec un spasme de l'hémiface et deux patients avec entropion en rapport avec l'âge. Le traitement a été efficace sur une période de 3 à 4 mois chez ces trois groupes de patients. À la reprise des symptômes, il s'est avéré nécessaire de procéder à une nouvelle chimio-dénervation au moyen de la toxine. La complication la plus fréquente a été une ptose transitoire chez $7.8 \%$ des cas. L'incidence de cette complication s'élève à $11.1 \%$ lorsqu'on utilise des doses de toxine supérieures à 25 unités pour chaque muscle orbiculaire. Le traitement a été bien accepté des patients et ils ont pu éventuellement revenir à leur mode de vie antérieur.

Can. J. Neurol. Sci. 1987; 14:42-45

Benign essential blepharospasm is a focal dystonia characterized by repeated, involuntary progressive squeezing of the eyelids, often so severe as to make the patient functionally blind. Initial symptoms may be ignored by the patient but the disease is however progressive. The lid closure may vary from one time to another although fatigue, anxiety or concentration on a task (eg., driving) may bring on an attack. Early on, patients may develop "tricks" to open the lids such as atonal whistling or pressing on the upper lip but later these tricks become ineffective and the patients may be unable to leave their homes unattended.

Lack of recognition of this disease due to its presumed rarity ( 1 in 10,000 patients who seek general medical care $)^{1}$ may cause the patients to consult faith healers, hypnotists, acupuncturists and chiropractors, as well as many physicians in an attempt to find a cure to what they are sure is not just a psychological problem.

Previous medical therapeutic options have included the use of systemic pharmacologic agents. 'These have limited effectiveness and may produce significant side-effects such as severe depression with tetrabenazine. ${ }^{2}$
Surgical procedures to weaken the effector muscle, the orbicularis oculi, have a long history. Fox in $1951^{3}$ first subtotally removed the upper eyelid orbicularis in a patient with blepharospasm following encephalitis. The procedure was expanded and modified by Gillam and Anderson in $1981^{4}$ in which subtotal, preseptal and pretarsal orbicularis extirpation in association with removal of the corrigator superciliaris and procerus muscles was performed bilaterally with a bilateral browplasty. Complications of this procedure however include corneal exposure, partial necrosis of skin in the upper lid, partial forehead anesthesia but there was a significant relief of blepharospasm in all patients treated.

Selective peripheral facial nerve ablation was first described by Reynolds and co-workers in $1967 .{ }^{5}$ It has been reported by Callahan ${ }^{6}$ that the two year recurrence rate for this procedure is 50 to $55 \%$.

The clinical application of the known potent muscle paralytic effect of botulinum toxin was pioneered by $\mathrm{Scott}^{7.8 .9}$ initially in the monkey and then in human extraocular muscles in the pharmacologic treatment of strabismus. The fact that there was no systemic effect from the toxin injections made the applica-

From the Department of Ophthalmology, University of British Columbia, Vancouver (Dr. Carruthers) and the Department of Biomedical and Environmental Health Science, School of Public Health, University of California, Berkeley (Dr. Stubbs)

Presented at the 48th Annual Meeting of the Canadian Ophthalmological Society, June 25, 1985, Toronto, Ontario

Received January 22, 1986. Accepted in final form September 17, 1986

Reprint requests to: Dr. Jean Carruthers, Suite 210, 2730 Commercial Drive, Vancouver, B.C., Canada V5N 5P4 
tion to other muscle groups an obvious extension. Although Clostridium botulinum produces six antigenically distinct exotoxins $A$ to $F$, only $A$ was used in our investigation. Its mechanism of action is to interfere with acetylcholine release from nerve terminals. In extrapolation from monkey data, the lethal dose for $50 \%$ survival in humans is estimated to be $2.0 \mu \mathrm{g} .{ }^{9}$

Botulinum toxin injection is performed in the outpatient clinic setting with no need for hospitalisation or general anesthesia. The denervation and symptomatic relief usually begins three days after injection and lasts for three to four months.

A multicenter control trial of botulinum toxin for the treatment of strabismus was started in 1982 from the Smith-Kettlewell Institute of Visual Sciences in San Francisco under the direction of Dr. Alan Scott. The use of botulinum toxin in the treatment of blepharospasm began one year later and is still in progress.

\section{Materials and Methods}

The botulinum A exotoxin* used in this study is prepared under the direction of the Smith-Kettlewell Institute of Visual Sciences in San Francisco so that all co-investigators use identically-prepared pharmacologic agent. The toxin is stored frozen at -4 degrees centrigrade and is removed from the deep freeze immediately prior to use. Sterile saline without preservative is used to dilute the toxin to the appropriate concentration and the required dose is drawn up in a tuberculin syringe. All first injections receive no more than 12.5 units of toxin per orbicularis oculi. Depending on clinical effect, subsequent doses may increase. The authors have not needed to use more than $\mathbf{5 0}$ units of toxin per orbicularis to date. No specialized equipment is otherwise needed.

All patients received a complete ophthalmologic examination including evaluation of their blepharospasm on the scale, (1) mild blinking excess, (2) noticeable blinking, (3) strong spasms with marked activity restriction, (4) incapacitation due to spasm. Their orbicularis force was also assessed subjectively by the examiner attempting to prevent the patient's forced eyelid closure on a 5-point scale with zero as no orbicularis power and four as extremely overactive orbicularis power. All patients were photographed before and one week after injection. Patients with hemifacial spasm were seen by a neurologist to attempt to identify those individuals who may have had a space-occupying lesion in the ipsilateral posterior fossa.

After full informed consent, patients were injected according to the experimental protocol directed by Dr. Alan Scott. The purified botulinum toxin was injected in five positions in the affected lid (see Figure 1). Spasm did not usually affect the temporal brow much and so this site was deleted from the original six injection positions. Injections in the upper lid were made with the needle pointing away from the levator aponeurosis. Patients should be injected while sitting or if injected supine, should have their head elevated immediately to reduce the chance of toxin diffusing through the orbital septum and producing ptosis by denervating the levator muscle.

Initial treatment was 12.5 units of purified botulinum toxin per eye and subsequent doses were titrated according to desired clinical effect. Many patients could last 12 to 16 weeks between

*Botulinum A exotoxin registered name Oculinum (R) available from Dr. Alan B. Scott, Smith-Kettlewell Institute of Visual Sciences, 2232 Webster Street, San Francisco, California injections on 12.5 units but others seemed to require higher doses. One man who weighed nearly $300 \mathrm{lbs}$ required 50 units per eye to produce acceptable results over the same period.

\section{RESULTS}

\section{(a) Benign Essential Btepharospasm (BEB)}

Twenty-seven women (57.4\%) and 20 men (42.6\%) were seen with this condition and treated with botulinum toxin injections (see Figure 2). Seventy percent of the men were aged 40 to 69

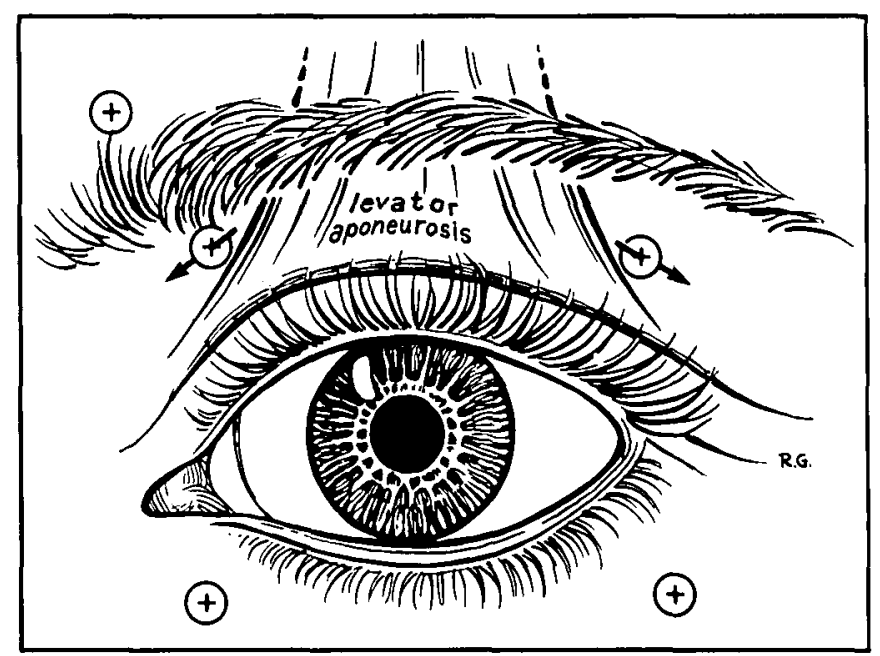

Figure 1 - Injection Sites for Blepharospasm. Note the needle tip is directed away from the levator aponeurosis in injection of the upper lid to prevent the complication of ptosis.
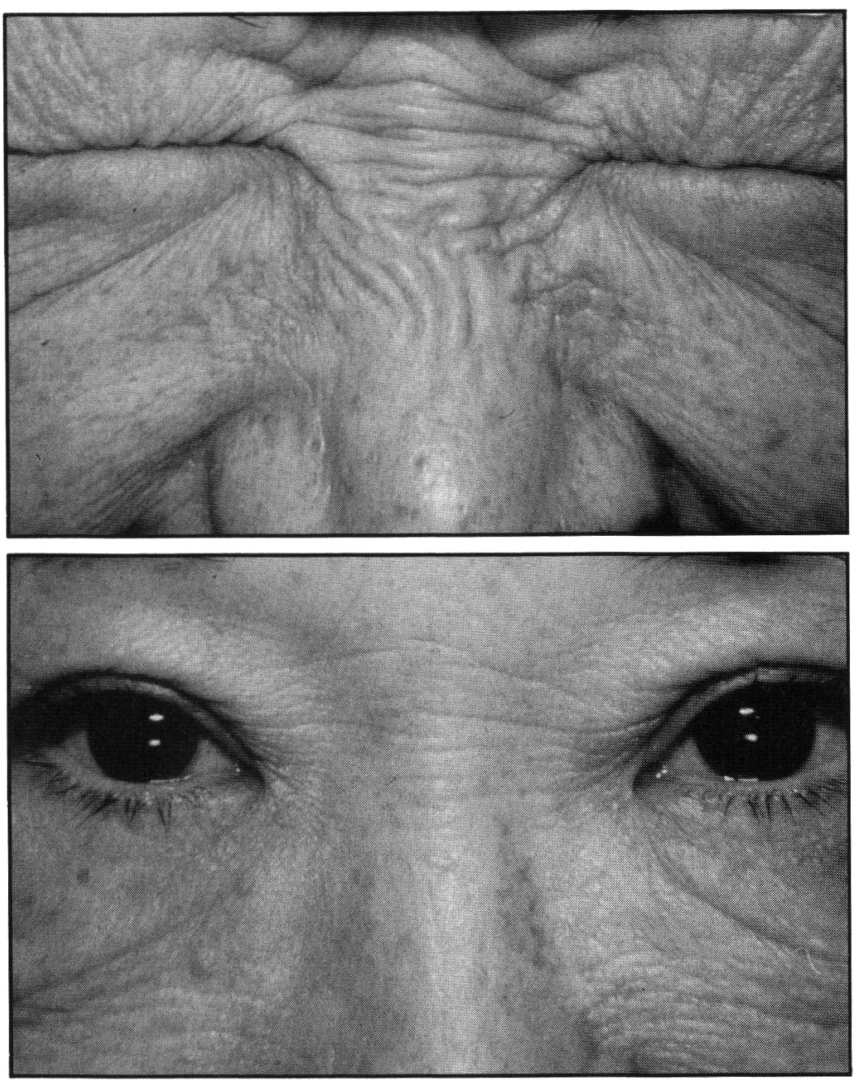

Figure 2 - Pre (above) and Post (below) injection appearance of a 64 year old female patient with bilateral essential blepharospasm. The "post" picture is taken one week after injection. 
years as were $81.5 \%$ of the women, suggesting that women tend to develop this condition later than men or seek medical assistance later.

Approximately one half $(56.5 \%)$ of these patients had the condition for less than 5 years at the time of first treatment with botulism toxin and nearly one third of the patients, $32.6 \%$, had the condition for at least 10 years. No patients had associated stroke, but $42.5 \%$ had associated cranial dystonia of Meige syndrome ${ }^{2}$ and $4.3 \%$ had Parkinson's disease with bilateral blepharospasm as a complication of their disease.

All the patients experienced subjective relief of spasm and excessive blinking within 2 to 3 days of each injection. They had a reduction in severity of their blepharospasm to either no blinking or spasm or to mild blinking excess only. Many patients reported that their best response was the initial one. On subsequent injections, the same or often a higher dose was needed to give a similar relief. Objective evaluation of orbicularis force showed zero power to resist forced opening of the lids by examiner within one week after the injection. Patients with severe spasm, $4+$, often needed to return several weeks after the initial injection for a repeat injection of 12.5 units of toxin. In general, large individuals required a larger dose of toxin for maintenance after initial assessment of their response to the starting dose than individuals of smaller mass. Patients of both sexes had a mean of 14.5 weeks between the first and second injections and of 16.9 weeks between the second and third injections. The interval between third and subsequent injections was 9.5 weeks but this figure was based on the artificial cut-off to analyze the results.

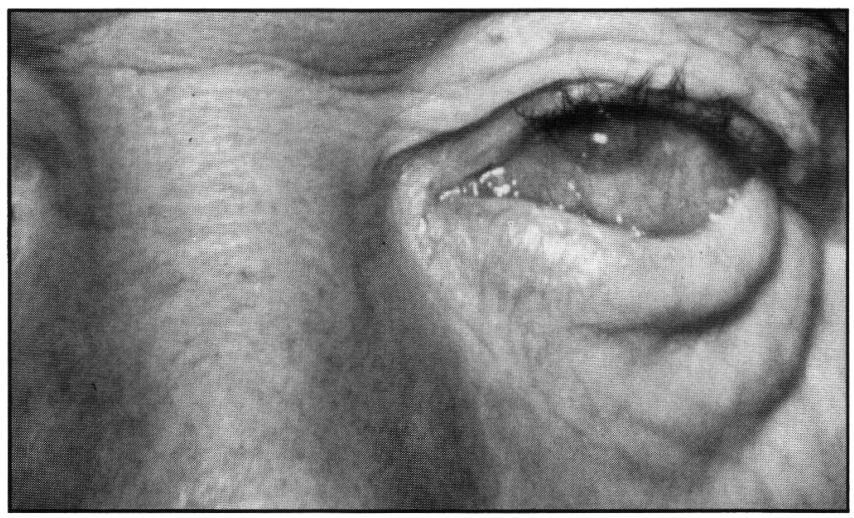

Figure 3 - Age-related entropion: pre-injection appearance of the left eye showing trichiasis, epiphora and injection.

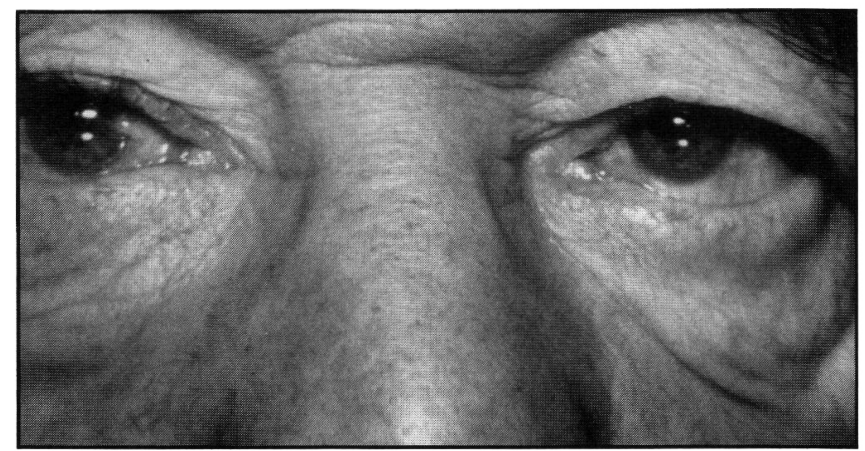

Figure 4 - Age-related entropion left eye showing resolved lower eyelid malposition with absent trichiasis and injection.

\section{(b) Hemifacial Spasm (HS)}

Four males and seven females were treated. The males were between 50 to 80 years of age and the females slightly older at 60 to 80 years. All had grading of their blepharospasm in the affected eye and orbicularis force on the same assessment protocol as for a bilateral blepharospasm. All received initial and subsequent doses of toxin as for the bilateral blepharospasm patients and they responded with a prompt reduction of orbicularis force as did the bilateral blepharospasm patients. They too responded for mean of 3 months before requiring reinjection because of recurrent spasm.

\section{(c) Age-Related (so-called 'Spastic') Entropion}

One patient (JW) had bilateral entropion with marked ocular discomfort due to his trichiasis (Figure 3 ). He had refused surgical repair. He received injections every 12 to 16 weeks bilaterally and was given only 5 units in two locations in each lower eyelid (Figure 4). After $1 \frac{1}{2}$ years of toxin injections and despite the complete remission between injections, he was persuaded to have surgical repair.

One man (DM) had bilateral spastic entropion in addition to his Parkinsonism and bilateral benign essential blepharospasm. His health was too fragile to consider surgery and his relief of entropion and spasm on an outpatient basis with botulinum toxin injections was symptomatically complete.

\section{COMPLiCATIONS}

\section{Blepharospasm/Hemifacial Spasm}

The main complication observed amongst these patients is transient ptosis. It is usually $1-2 \mathrm{~mm}$ and subsides in one to six weeks. It was seen in $5.1 \%$ of patients receiving less than 25 units and $11.7 \%$ of patients receiving more than 25 units of toxin per eye. It did not seem to increase in incidence with subsequent injection although it appeared to increase with higher doses of toxin (Table 1).

One patient with hemifacial spasm sustained an ipsilateral ptosis and 4 prism diopter right hypotropia after 12.5 units or purified botulism toxin in the right orbicularis. The hypotropia was treated with Fresnel prisms and recovered in 9 weeks. Presumably the age-related fenestrations in the orbital septum

Table 1: Occurrence of Ptosis per Eye Injected by Dose per Eye and Injection Number for Essential Blepharospasm

\begin{tabular}{ccr}
\hline \hline \multirow{2}{*}{$\begin{array}{c}\text { Dose/Eye } \\
\text { (units) }\end{array}$} & \multicolumn{2}{c}{$\begin{array}{c}\text { PTOSIS } \\
\text { Yes }\end{array}$} \\
\cline { 2 - 3 }$<25$ & Freq & $\%$ \\
\hline 25 & 7 & 5.1 \\
\hline Total & 11 & 11.7 \\
\hline y & 18 & 7.8 \\
\hline
\end{tabular}

$\mathrm{X}^{2}=3.7, \mathrm{p}<0.10$

\begin{tabular}{ccc} 
& \multicolumn{2}{c}{$\begin{array}{c}\text { PTOSIS } \\
\text { Injection }\end{array}$} \\
\cline { 2 - 3 } Number & Freq & $\%$ \\
\hline 1 & 8 & 8.2 \\
2 & 5 & 7.6 \\
$3+$ & 5 & 7.4 \\
\hline Total & 18 & 7.8 \\
\hline
\end{tabular}

$X^{2}=0.04, p>0.9$ 
allow toxin migration to the levator as well as to the superior rectus.

Some patients experienced transient tearing over 1-2 weeks post-injection. Others with reduced tear production reported a transient exacerbation of their dry eye syndrome, easily controlled with topical ocular lubrication.

\section{Age-related lower eyelid entropion}

There were no severe complications seen in this group. There was no lower eyelid ptosis and the only problem was recurrence of the entropion at 3-4 monthly intervals, as with the blepharospasm patients. (Figures 3-4)

\section{DisCUSSION AND CONCLUSIONS}

Botulinum toxin has been used for over three years for the treatment of benign essential blepharospasm, hemifacial spasm and their variants. Alternative modes of therapy for these conditions include the use of many centrally-acting drugs ${ }^{3}$ that are dopaminergic antagonists or cholinergic agonists but most patients fail to gain long-term relief from these medications. Surgical alternatives for treatment of these conditions include orbicularis oculi stripping as described by Anderson and selective seventh nerve avulsion as described by Reynolds. In a comparison of the two techniques and two matched series of twenty-two patients, McCord and co-workers found that secondary procedures were needed almost five times as often with the Reynolds procedure as with the Anderson procedure and that patient acceptance was much greater with the latter procedure. ${ }^{10}$ Thus the end organ approach of muscle extirpation is more effective than nerve ablation because of the reduced recurrence risk and unlike botulinum toxin injection, it is permanent. However, botulinum toxin injections are not complicated by forehead anesthesia, permanent lagophthalmos in tear-deficient patients or lid ischemia.

The subcutaneous periocular injection of botulinum toxin is another means to produce a muscle extirpation. The botulinum toxin injections temporarily relieve the spasm in most patients. The interval of relief is variable but is usually approximately three months. The local side-effects were well tolerated by our patients and no patient showed systemic side-effects.

The vertical diplopia and ptosis experienced in one patient with hemifacial spasm was treated conservatively with a Fresnel prism on the spectacle lens and remitted in nine weeks. Presumably toxin migrated through the orbital septum fenestrations to the levator muscle and the subjacent superior rectus motor endplate. This patient has since had subsequent botulinum injections without this complication.

The injection of botulinum toxin provides safe effective relief of blepharospasm in bilateral and unilateral patients. " It is also effective in treating age-related entropion. Patients with spasmodic torticollis also show temporary symptomatic relief from the botulinum injections. It is possible that with repeated denervation, the interval of relief may extend so that there may be some permanent effect from the injections in all muscle groups treated.

\section{REFERENCES}

1. Henderson JW. Essential blepharospasm. Tr Am Ophthal Soc 1956; 54: 453-520.

2. Bird AC, McDonald WI. Essential blepharospasm. Tr Ophthalmol Soc U.K. 1975: 95: 250-253.

3. Fox SA. Relief of int ractable blepharospasm, a preliminary report. Am J Ophthalmol 1951; 34: 1351-1356.

4. Gillam WN, Anderson RL. Blepharospasm surgery. Arch Ophthalmol 1981: 99: 1056-1062.

5. Reynolds DH, Smith JL. Walsh TJ. Differential section of the facial nerve for blepharospasm. Trans Am Acad Ophthalmol Otolaryngol 1967; 71: 656-664.

6. Callahan A. Intractable blepharospasm. Am J Ophthalmol 1965; 60: 788-791.

7. Scott AB, Rosenbaum AL. Collins CL. Pharmacologic weakening of extraocular muscles. Invest Ophthalmol 1973: 12: 924-927.

8. Scott AB. Botulinum toxin injection into extraocular muscles as an alternative to strabismus surgery. Ophthalmology 1980; 10: 1044-1049.

9. Scott AB. Botulinum toxin injection of eye muscles to correct strabismus. Trans Am Ophthalmol Soc. 1981; 79: 734-770.

10. McCord CP, Coles WH, Shore JW, Spector R, Putnam J. Treatment of essential blepharospasm I. Comparison of facial nerve avulsion \& eyebrow-eyelid muscle stripping procedure. Arch Ophthalmol 1984; 102: 266-268.

11. Scott AB, Kennedy RA. Stubbs HA. Botulinum toxin injection as a treatment for blepharospasm. Arch Ophthalmol 1985: 103: 347-350.

12. Tsui JK, Eisen A, Mak E, Carruthers J, Scott AB, Calne DB. A pilot study on the use of botulinum toxin in spasmodic torticollis. Can J Neurol Sci 1985; 4: 314-316. 